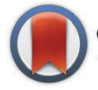

CrossMark \& click for updates

Cite this: Phys. Chem. Chem. Phys., 2015, 17, 10004

Received 8th January 2015, Accepted 9th March 2015

DOI: $10.1039 / c 5 c p 00112 a$

www.rsc.org/pccp

\section{Zinc oxide as a defect-dominated material in thin films for photovoltaic applications - experimental determination of defect levels, quantification of composition, and construction of band diagram}

\author{
Maciej Krzywiecki, ${ }^{\text {ab }}$ Lucyna Grządziel, ${ }^{\mathrm{b}}$ Adnan Sarfraz, ${ }^{a}$ Danish Iqbal, ${ }^{a}$ \\ Anna Szwajca ${ }^{c}$ and Andreas Erbe ${ }^{a}$
}

\begin{abstract}
In the present work, thin ZnO layers were synthesized by the sol-gel method with subsequent spin-coating on $\mathrm{Si}(100)$. We show that the detailed analysis of lab-recorded photoemission spectra in combination with Kelvin probe data yielded the work function, ionization energy, and valence band - Fermi level separation - and hence enabled the construction of band diagrams of the examined layers. With small modifications in preparation, very different films can be obtained. One set shows a homogeneous depth-dependent $\mathrm{n}$ carrier distribution, and another a significant carrier concentration gradient from $\mathrm{n}$-type conductivity to almost metal-like $\mathrm{n}^{+}$character. Likewise, the surface morphology can be tuned from a uniform, compact surface with spherical single-nm sized grain-like features to a structured surface with 5-10 nm tall crystallites with (002) dominating crystal orientation. Based on the band-bending and the energy levels observed, defects of contradictory nature, i.e. acceptordonor-trap (ADT) properties, were identified. These defects may be groups of point defects, with opposite character. The ADT states affect the energy levels of the oxide layers and due to their nature cannot be considered in the photoemission experiment as mutually independent. The versatile nature of the synthesis provides us with the opportunity to tune the properties with a high degree of freedom, at low processing costs, yielding layers with an exotic electronic structure. Such layers are interesting candidates for applications in photovoltaic and nanoelectronic devices.
\end{abstract}

\section{Introduction}

$\mathrm{ZnO}$ is a wide-gap semiconductor with optical transparency in the visible range. The optical and electronic properties of $\mathrm{ZnO}$ thin films resulted in applications in electronic and optoelectronic devices such as transparent conductors, ${ }^{1-3}$ solar cell windows, ${ }^{4-6}$ ultraviolet lasers, ${ }^{7}$ gas sensors, ${ }^{8,9}$ refractive index sensors,${ }^{10}$ light emitting diodes ${ }^{11,12}$ and surface acoustic wave devices. ${ }^{13}$ As n-type semiconductors, ZnO layers could also be a good candidate for dye-synthesized solar cells ${ }^{14}$ or for use in hybrid inorganic-organic electronic devices. ${ }^{15,16}$

Due to the vast range of applications, numerous $\mathrm{ZnO}$ film preparation techniques have been employed, such as chemical vapor deposition, ${ }^{17,18}$ ion beam deposition, ${ }^{19}$ molecular beam epitaxy, ${ }^{20}$ magnetron sputtering, ${ }^{21-23}$ atomic layer deposition ${ }^{24}$

\footnotetext{
${ }^{a}$ Max-Planck-Institut für Eisenforschung GmbH, Max-Planck-Str. 1, 40237 Düsseldorf, Germany

${ }^{b}$ Institute of Physics - CSE, Silesian University of Technology, Konarskiego 22B, 44-100 Gliwice, Poland. E-mail: Maciej.Krzywiecki@polsl.pl;

Fax: +48 (32) 23722 16; Tel: +48 (32) 2372001

${ }^{c}$ Faculty of Chemistry, A. Mickiewicz University, Umultowska 89b, 61-614 Poznań, Poland
}

or laser pulse deposition. ${ }^{25}$ All the above-mentioned methods resulted in well-established $\mathrm{ZnO}$ films with structure and properties more or less tuned to the desired application. However, the disadvantage of these methods is that they require advanced expensive setups.

Alternatives include electrochemical growth, ${ }^{26}$ or corrosion processes. ${ }^{27}$ Another relatively cheap method is based on a sol-gel process. Particularly studies of colloidal $\mathrm{ZnO}$ nanocrystals were extensively performed previously in terms of the crystallite size and luminescence-controllable synthesis. ${ }^{28,29}$ Only few studies have been recently devoted to the optical and structural characterization of the sol-gel derived $\mathrm{ZnO}$ thin films. In most cases the sol-gel technique is followed by another uncomplicated process which is spin-coat deposition. ${ }^{30-32}$ The authors focused on the influence of the growth conditions on the physical properties of ZnO thin films. Hence, a number of questions arose together with the expansion of knowledge on this topic. The electronic structure considerations or problems of ambipolar doping are discussed only in chosen studies (e.g. ref. 11 and 12). Nevertheless the zinc oxide thin film energy structure is still not fully revealed. Especially for photovoltaic applications, the mutual influence of the morphology and chemical composition on the electronic properties is crucial. 
For instance, understanding the role of native point defects (like vacancies) or the nature of impurities is a milestone on the way to control the properties (e.g. conductivity) of ZnO layers. ${ }^{33}$ By controlling the properties of the material one can easily tune the performance of the device under development; hence a deeper understanding of the $\mathrm{ZnO}$ internal structure is essential. $^{34}$

In the present work we focus on the comprehensive characterization of $\mathrm{ZnO}$ layers, obtained by a sol-gel technique, designed for bulk heterojunction inorganic-organic excitonic solar cells. The intended application requires a surface morphology such that in combination with phthalocyanine-based organic semiconductors, the $\mathrm{ZnO}$ layer should create a bulk heterojunction. According to our last studies on copper and iron phthalocyanines, ${ }^{35,36}$ the surface should be moderately developed for such applications, with roughness in the range of a few $\mathrm{nm}$ in such a manner that the incoming organic molecules could easily penetrate the outer ZnO surface creating a bulk interdigitated structure. Because of the exciton mean free path (which ranges from $\sim 7 \mathrm{~nm}$ up to $\sim 30 \mathrm{~nm}$ depending on the material ${ }^{37}$ ), the overall thickness of such a created $\mathrm{p}-\mathrm{n}$ junction should not exceed $15-30 \mathrm{~nm}$.

The main aim of this work is to use laboratory-based photoemission techniques to construct a band diagram of $\mathrm{ZnO}$ sol-gel layers. Furthermore, information on the morphology is required, which is obtained by scanning probe and scanning electron microscopy.

\section{Experimental details}

\subsection{Sample preparation}

Silicon(100) wafers (SiMat) (n-type, p-doped, 5-10 $\Omega \mathrm{cm}$ ) were used as substrates. The wafers were cleaned in an ultrasonic bath by sequentially soaking them in acetone, isopropanol, and de-ionized water for $15 \mathrm{~min}$ per cycle. Then, the wafers were blown with nitrogen and dried in a furnace at $110{ }^{\circ} \mathrm{C}$ for $30 \mathrm{~min}$.

ZnO sol was synthesized by dissolving zinc acetate in ethylene glycol at 0.5 M. The solution was stirred and heated to $70{ }^{\circ} \mathrm{C}$. Then, ethanolamine (MEA) was added drop-wise. The sol was further stirred at $70{ }^{\circ} \mathrm{C}$ for $30 \mathrm{~min}$ and left for aging for $12 \mathrm{~h}$. All reagents were purchased from VWR and used as received.

Spin-coat deposition (spin-coater P6700, Specialty Coating Systems Inc.) was done in cycles. Spinning was conducted at $500 \mathrm{rpm}$ for $2 \mathrm{~s}, 2000 \mathrm{rpm}$ for $1 \mathrm{~s}$ and $6000 \mathrm{rpm}$ for $29 \mathrm{~s}$. Before spinning, the entire substrate surface was covered with the solution. After deposition the samples were dried in air for $10 \mathrm{~min}$ at $110{ }^{\circ} \mathrm{C}$. After the desired number of cycles, samples were annealed in a tube furnace at $350{ }^{\circ} \mathrm{C}$ for $4 \mathrm{~h}$ in an ambient atmosphere.

Within this work, we report experiments conducted with samples after four deposition cycles (denoted as " $4 \mathrm{c}$ "), and samples after a single cycle (denoted as " $1 \mathrm{c}$ ").

\subsection{Experimental techniques}

Surface topography was investigated by atomic force microscopy (AFM) and scanning electron microscopy (SEM). Non-contact mode AFM investigations were done on a VEECO Nanoscope IV using Al-coated Olympus OMCL-AC160TS-R3 tips (resonant frequency $300 \mathrm{kHz}$, force constant $2.1 \mathrm{~N} \mathrm{~m}^{-1}$ ). AFM data were quantified using Gwyddion. The images were leveled and filtered. Quantitative analysis used Gwyddion built-in algorithms. ${ }^{38}$

SEM images were taken using a Carl Zeiss LEO 1550 VP microscope combined with an in-lens detector (working distance $3 \mathrm{~mm}$, ETH voltage $10 \mathrm{kV}$ ). For elemental analysis and basic chemical characterization of the sample, energy-dispersive X-ray spectroscopy (EDX; Oxford Instruments) was applied, with a setup integrated into the SEM.

X-Ray diffraction (XRD) was conducted using a Bruker-AXS D8 with a $\mathrm{Cu}-\mathrm{K} \alpha$ source.

Surface chemical composition was examined using X-ray photoemission spectroscopy (XPS), combined with $\mathrm{Ar}^{+}$ion etching steps for depth profiling on a Physical Electronics PHI Quantera II spectrometer equipped with an Al- $\mathrm{K}_{\alpha}$ microfocused (using a quartz crystal monochromator) source with a photon energy of $1486.74 \mathrm{eV}$ and a dual-beam charge neutralizer. The pass energy was set to $140 \mathrm{eV}$ for the survey spectra (energy step $0.2 \mathrm{eV}$ ) and $26 \mathrm{eV}$ for recording the individual core level spectra (energy step $0.05 \mathrm{eV}$ ). The XPS system base pressure was $2 \times 10^{-8} \mathrm{~Pa}$. All XPS spectra were recorded at a take-off angle of $45^{\circ}$.

The etching rate (spot size $2 \mu \mathrm{m} \times 2 \mu \mathrm{m} ; U=1 \mathrm{kV}$ ) was kept at $3.7 \mathrm{~nm} \mathrm{~min}{ }^{-1}$. The binding energy was calibrated to $\mathrm{Au} 4 \mathrm{f}_{7 / 2}$ $(84.0 \mathrm{eV}) .^{39}$

XPS data were analysed by curve fitting using CASA XPS software. Each peak was represented by a sum of Gaussian $(70 \%)$ and Lorentzian (30\%) lines, while the secondary electron background was subtracted utilizing the Shirley function. Where necessary, peaks were resolved with spin-orbit multiplets. The full width at half maximum (FWHM) of the same components was allowed to vary within a narrow range. We used the lowest possible number of components to fit the data satisfactorily, i.e. to obtain acceptably low residual values. The estimated uncertainty for particular component position determination in XPS measurements was within $0.05 \mathrm{eV}$. Quantitative analysis, including component ratio determination, was done using CASA XPS embedded relative sensitivity factors (RSF) and algorithms.

Ultraviolet photoemission spectroscopy (UPS) measurements were done using the SPECS PHOIBOS 150 analyzer. Excitation was performed using the $21.22 \mathrm{eV}$ He I line of a He discharge lamp. He I $\beta$ and He I $\gamma$ satellites were numerically subtracted from the recorded spectra. ${ }^{40}$ The estimated uncertainty was $0.05 \mathrm{eV}$ for the obtained work function and $0.07 \mathrm{eV}$ for the ionization energy.

Spectroscopic ellipsometry was used to estimate the deposited zinc oxide thickness. Measurements were conducted using a Sentech Instruments SE 800 spectroscopic ellipsometer working in the wavelength range $350-810 \mathrm{~nm}(1.55-3.55 \mathrm{eV})$ at angles of incidence from $55^{\circ}$ to $70^{\circ}$ in $5^{\circ}$ steps. Analysis was performed using the instrument's software, using a Si substrate/Si oxide/ZnO/air multilayer Cauchy model with parameters implemented in the software. The silicon oxide thickness was determined before deposition of $\mathrm{ZnO}$ to be $2.5 \mathrm{~nm}$.

Surface potential scans were performed on a commercial scanning Kelvin probe (SKP) system (Wicinski - Wicinski GbR, Wuppertal, Germany) with a $100 \mu \mathrm{m}$ NiCr tip in a dry nitrogen 
atmosphere. Before experiments, the probe potential was calibrated against a $\mathrm{Cu} / \mathrm{CuSO}_{4}$ reference and against Au plates.

\section{Results and discussion}

\subsection{Surface topography and structure}

Before proceeding to a detailed electronic structure determination of the $\mathrm{ZnO}$ films, the films where characterized by a number of analytical techniques.

Ellipsometric determination of the layer thickness yields values

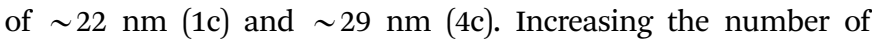
deposition cycles hence increases the thickness only slightly, due to dissolution of the previously deposited sol before annealing.

AFM measurements (Fig. 1) of the annealed ZnO layers show the surface with uniformly distributed small spherical grains. The estimated RMS surface roughness is $1.4 \mathrm{~nm}$ (1c) and $2.8 \mathrm{~nm}(4 \mathrm{c})$, hence it increases with increasing film thickness. The roughness of the Si wafer substrate was $<0.4 \mathrm{~nm}$. Fig. 1 clearly exhibits that an increase in the number of deposition cycles is accompanied by an increase in the grain diameter. The control experiment conducted for silicon substrates revealed the RMS roughness to be below $0.25 \mathrm{~nm}$; hence it should not have an impact on the layers' topography.

The estimated grain diameter determined from profiles is 25-30 $\mathrm{nm}$ for sample $1 \mathrm{c}$ and 50-75 $\mathrm{nm}$ for sample 4c (Fig. 1c and $\mathrm{d}$, respectively). An interesting feature present in the $4 \mathrm{c}$ ZnO layer is uniformly distributed "column-like" crystallites protruding from the sample surface. Their height is estimated to be 10-15 $\mathrm{nm}$. Such protruding crystallites are not visible for thinner samples. The grain coarsening with increasing number of deposition cycles is confirmed by SEM images shown in Fig. 2.

The SEM images also confirm the existence of "column-like" crystallites in the case of $4 \mathrm{c}$ samples, and a more homogenous surface with a uniform crystallite distribution in the case of the 1c sample. The "column-like" structure is a direct consequence of the partial dissolution of the previously deposited sol layer for multiple deposition cycles.

XRD measurements (Fig. 3) show two strong reflections at $56.2^{\circ}$ and at $34.5^{\circ}$. The first one can be attributed to some amorphous compounds that did not convert to crystalline $\mathrm{ZnO}$ or to the (311) base silicon substrate ${ }^{41}$ while the latter is the reflection originating from $\mathrm{ZnO}(002)$ crystallites. ${ }^{42}$
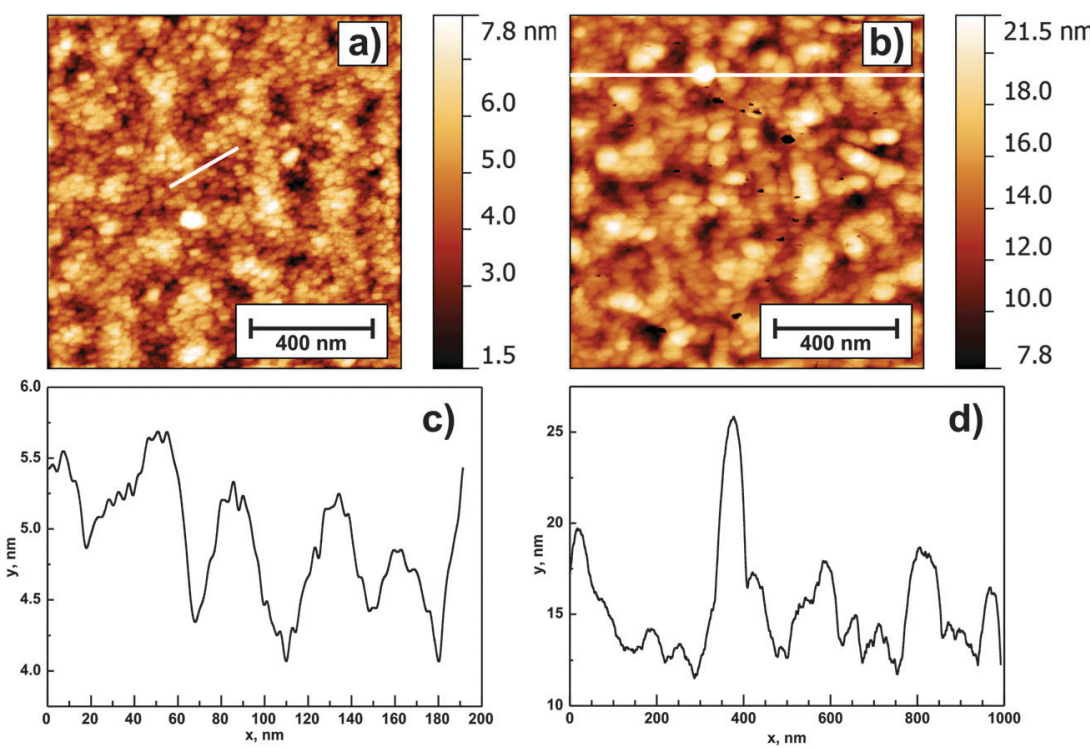

Fig. 1 AFM images of $1 \mu \mathrm{m} \times 1 \mu \mathrm{m}$ height obtained for samples $1 \mathrm{c}(\mathrm{a})$ and $4 \mathrm{c}$ (b). Panels (c) and (d) show the extracted profiles marked with white lines in the corresponding images.
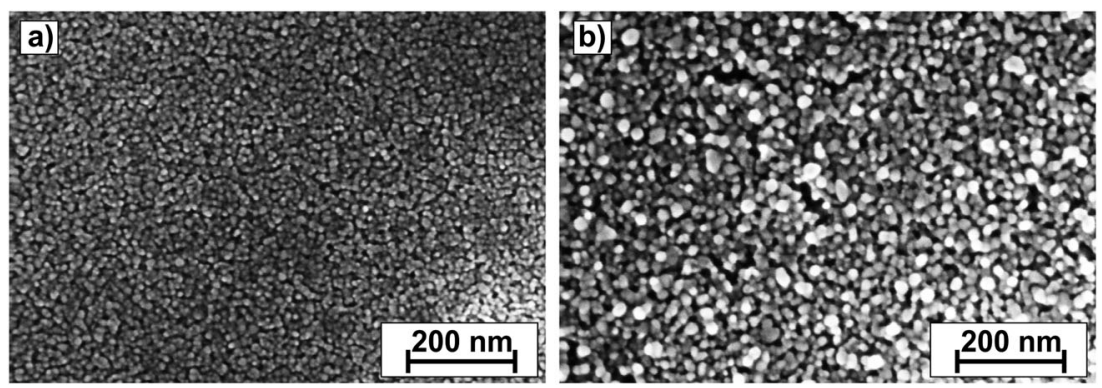

Fig. 2 SEM images of samples $1 c(a)$ and $4 c(b)$. 


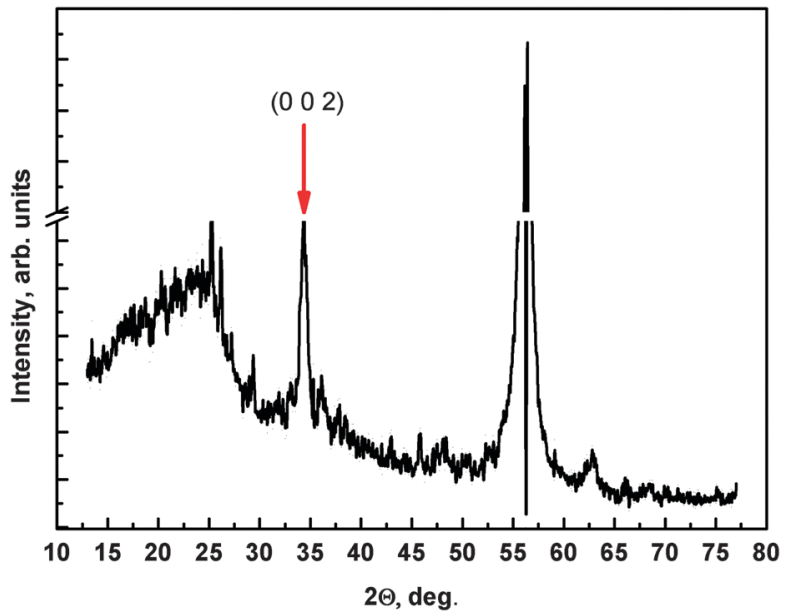

Fig. 3 XRD pattern of the $4 c$ sample. The peak at about $56^{\circ}$ is a peak also present in reference measurements.

Scherrer's formula ${ }^{43}$

$$
L=\frac{K_{\mathrm{s}} \lambda}{\beta^{\prime} \cos \theta}
$$

was used for estimation of the volume mean crystallite size. In the above formula, $L$ is the mean crystallite diameter, $K_{\mathrm{s}}$ a shape-dependent constant (considered as close to unity, here $\left.K_{\mathrm{s}}=0.9^{42}\right), \lambda$ the X-ray wavelength $(0.154 \mathrm{~nm}), \beta^{\prime}$ the full width at half maximum (FWHM) of the peak under consideration (in radians) and $\theta$ the corresponding Bragg angle. The estimated volume crystallite size in the case of the layers ranges from $10 \mathrm{~nm}$ for sample $1 \mathrm{c}$ to $15 \mathrm{~nm}$ for sample $4 \mathrm{c}$. In agreement with microscopic experiments, the crystallite size is larger for the sample with higher number of deposition cycles. Actual numbers derived from XRD are lower compared to AFM, because of the different averaging.

As a result, application of several sol deposition cycles leads to roughening of the surface because of an increase in individual grain size, at a moderate increase in layer thickness. At the same time, modification of the overall morphology is observed.

\subsection{Photoemission studies}

3.2.1 Chemical composition analysis. Application of XPS stepwise ion etching allowed for the determination of surface and depth-dependent chemical composition of examined $\mathrm{ZnO}$ layers. Fig. 4 shows survey spectra recorded for several chosen steps of ion etching. ZnO-related energy regions are well visible, i.e. $\mathrm{Zn}(2 \mathrm{p}, 3 \mathrm{~s}, 3 \mathrm{p}, 3 \mathrm{~d}), \mathrm{Zn} \mathrm{LMM}$ and $\mathrm{O}$ 1s. Carbon contamination can be monitored in the $\mathrm{C} 1 \mathrm{~s}$ region. With increasing depth, the substrate peaks ( $\mathrm{Si} 2 \mathrm{p}$ and $\mathrm{Si} 2 \mathrm{~s}$ ) become more pronounced, while those originating from ZnO diminish. The XPS spectral line assignment was based on the LaSurface ${ }^{44}$ database.

Carbon could be present in the samples as residuals from the preparation, or as a surface contaminant. C 1s energy region analysis (not shown) revealed that over $90 \%$ of carbon contamination is present on the sample's surface. In the interior of the film only trace amounts of carbon are detectable.

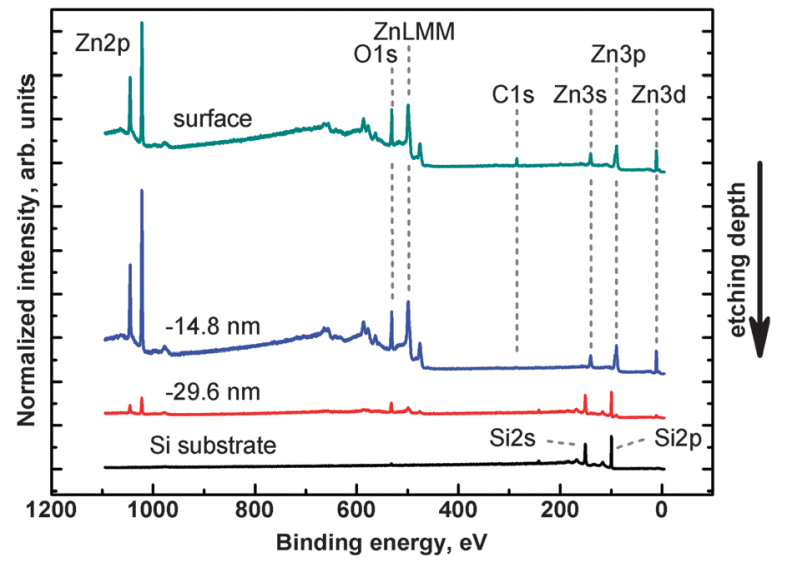

Fig. 4 Survey spectra obtained for the as-deposited 4c sample (surface) and three chosen steps of ion etching. All characteristic XPS regions are clearly visible.

This indicates that the prolonged annealing process is sufficient to remove the residual solvent from the film. Also substrate surface carbon contamination does not play a significant role as analysed in substrate XPS control measurement before $\mathrm{ZnO}$ layer deposition.

The chemical state of the examined oxide layer is examined in two ways. First, the Auger parameter $\alpha$ is evaluated and analyzed. ${ }^{45}$ Secondly, standard main oxygen (O 1s) and zinc (Zn 2p) regions are analyzed quantitatively. The evaluation of the Auger parameter $\alpha$ involves a method in which the differences in bonding negativity according to Pauling theory ${ }^{46}$ are calculated. The chemical state of examined species is determined from the energy difference between a representative XPS peak and a suitable Auger peak. The Auger parameter $\alpha$ is defined as: ${ }^{47}$

$$
\alpha=E_{\mathrm{k}}(j k l)+E_{\mathrm{B}}(i)
$$

where $E_{\mathrm{K}}(j k l)$ is the kinetic energy of the Auger transition $j k l$ and $E_{\mathrm{B}}(i)$ is the binding energy of an electron at atomic level $i$. In our case, the Zn $2 \mathrm{p}_{3 / 2}$ and $\mathrm{Zn}$ LMM should be considered. A lower $\alpha$ indicates a lower electron density at the atom, i.e. a higher oxidation state. Fig. 5 shows the $\alpha$-parameter of the $4 \mathrm{c}$ sample determined for different ion etching depths. The inset presents an example of the $\mathrm{Zn}$ LMM Auger peak for the non-etched $4 \mathrm{c}$ sample surface.

The general trend of the data is marked by the fitted polynomial line. It is easy to see that there are significant differences between the surface layer and the film. A higher oxidation state near the surface is expected, as the surface is in contact with the ambient atmosphere in the annealing and also prior to XPS measurements. A decrease of the $\alpha$-parameter is also detected near the $\mathrm{ZnO} /$ substrate interface. This decrease may originate from the influence of the substrate oxide film. ${ }^{48}$ More details can be obtained from an analysis of the $\mathrm{O} 1 \mathrm{~s}$ and $\mathrm{Zn} 2 \mathrm{p}$ regions.

Fig. 6a presents the $\mathrm{O} 1 \mathrm{~s}$ intensity as a function of etching depth. The overall peak consists of at least three components, from which only two are detectable at one time. The inset of Fig. 6a shows two components of the $\mathrm{O} 1$ s peak recorded for the 


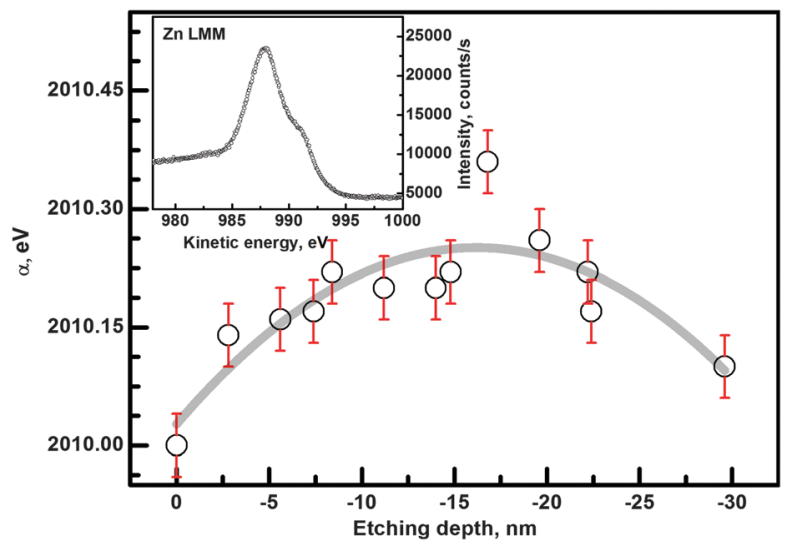

Fig. 5 Determined $\alpha$-parameter as a function of ion etching depth for the 4c sample. The depth dependence was fitted with a polynomial to show the trend in the data. The inset shows the Auger Zn LMM energy region.

4c sample etched to $\sim 16.8 \mathrm{~nm}$. Two layer-related components at $\sim 531 \mathrm{eV}$ and $\sim 532.5 \mathrm{eV}$ are well visible in the spectra and can be attributed, respectively, to oxygen-metal (here: $\mathrm{Zn}-\mathrm{O}$ ) bonding and probably to oxygen on a split-interstitial configuration $^{49}$ or oxide near $\mathrm{Zn}$ vacancies. The third component $(\sim 532 \mathrm{eV})$ appears after $\sim 30 \mathrm{~nm}$ of ion etching and hence originates from the substrate oxide. The initial increase in the O 1s signal after the first etching cycle is attributed to the removal of carbon contamination from the sample surface.

Quantification of the $\mathrm{Zn} 2 \mathrm{p}$ region allowed the determination of the $\mathrm{Zn} / \mathrm{O}$ concentration ratio, which is shown in Fig. $6 \mathrm{~b}$.

The data presented in Fig. $6 \mathrm{~b}$ for sample $4 \mathrm{c}$ show that the $\mathrm{Zn} / \mathrm{O}$ ratio increases from the value of 1.7 up to 2.1 (estimation uncertainty 0.06 ) at $\sim 10 \mathrm{~nm}$ depth, then it stabilizes up to $\sim 18 \mathrm{~nm}$ and then it decreases monotonically until the $\mathrm{ZnO} /$ substrate interface. For the $1 \mathrm{c}$ sample, the $\mathrm{Zn} / \mathrm{O}$ ratio starts at $\sim 1.80$ at the surface, and slightly increases to $\sim 2.00$ for a depth less than $3 \mathrm{~nm}$. It remains stable up to $\sim 14 \mathrm{~nm}$ and monotonically decreases similar to the $4 \mathrm{c}$ sample.

The difference in the $\mathrm{Zn} / \mathrm{O}$ ratio can be attributed to more pronounced surface oxidation during the annealing process after deposition in the case of $4 \mathrm{c}$ samples, because $4 \mathrm{c}$ samples exhibit a more developed surface topography in comparison to densely packed crystallites in 1c samples. The more "open" structure of $4 \mathrm{c}$ samples favors oxygen uptake from the atmosphere. A second contribution may be the partial dissolution of the previously deposited layer when applying several layers consecutively, thus resulting in a gradient of the $\mathrm{Zn}$ concentration. The decrease of the $\mathrm{Zn} / \mathrm{O}$ ratio towards the substrate is caused by substrate contributions and an overall decrease in intensity of $\mathrm{O} 1 \mathrm{~s}$ and $\mathrm{Zn} 2 \mathrm{p}$.

Overall, an atomic $\mathrm{Zn} / \mathrm{O}$ ratio of $\sim 2$ is obtained in the center of the film, which is far from the expected value of 1 for stoichiometric ZnO. This observed difference can only be explained by defects, and will be discussed in Section 3.2.2.

3.2.2 Electronic structure of the $\mathrm{ZnO}$ films. Detailed analysis of the valence band and $\mathrm{Zn} 3 \mathrm{~d}$ energy region (0-15 eV binding energy) of the XP spectra and additional application of UPS allowed for the description of the $\mathrm{ZnO}$ electronic properties. Because the ion

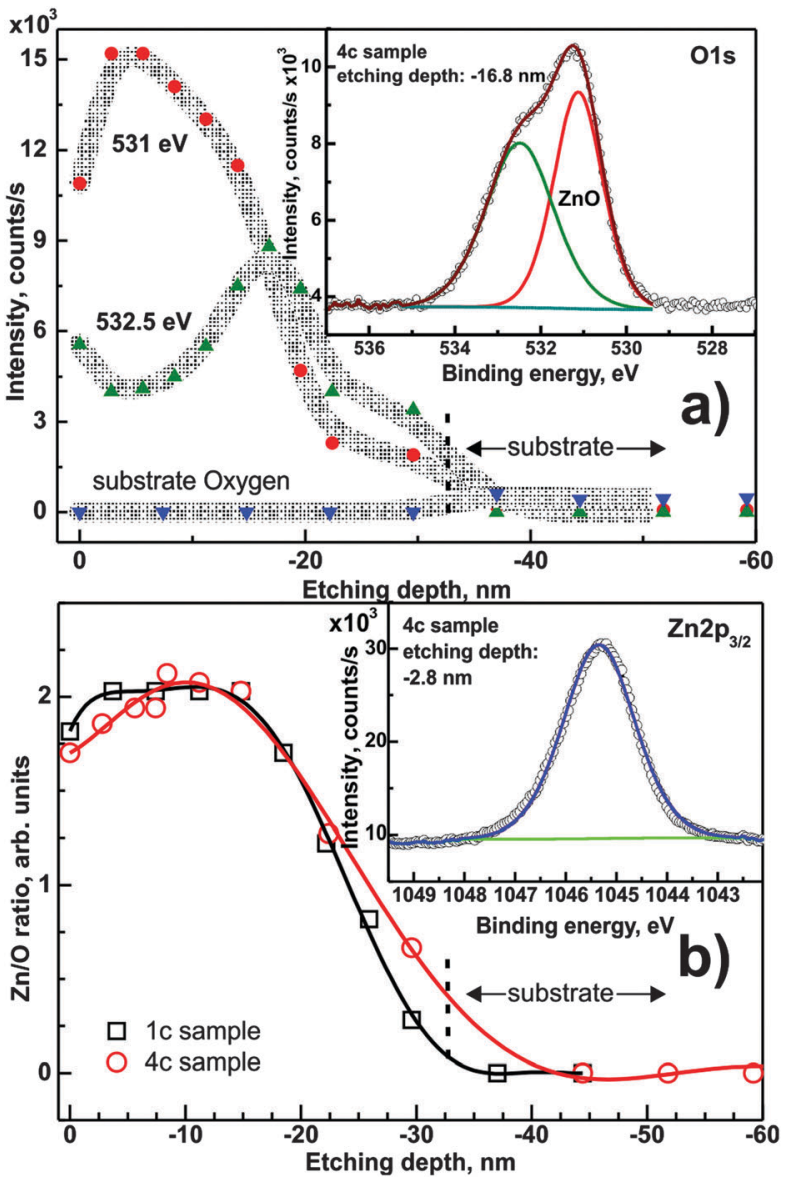

Fig. 6 (a) Components of the 01 XPS region as a function of the depth of ion etching for the $4 \mathrm{c}$ sample. Inset: decomposition of the $\mathrm{O} 1 \mathrm{~s}$ peak recorded for the $16.8 \mathrm{~nm}$ etched sample. Grey lines are eye-guiding spline connections. (b) The $\mathrm{Zn} / \mathrm{O}$ ratio as a function of the depth of ion etching for samples $1 c$ and $4 c$. Inset: the $Z n 2 p_{3 / 2}$ XPS peak. Lines between points are polynomial fits.

etching depth profile was obtained only for XPS, the UPS information should be treated here as a starting point for relating exactly XPS energies to the Fermi level. XPS and UPS have different depth of information, because of different photoelectron escape depths for different excitation energies. According to the Seah-Dench diagram ${ }^{50}$ while the UPS yields a photoemission signal from a few nanometers, XPS gives information down to $10 \mathrm{~nm}$. Hence, different surface and layer's interior species contribute differently to the overall signal.

Considering that Fermi levels of the analyzer and the investigated sample are equal, one can determine the energy difference between the Fermi level and the valence band onset $E_{\mathrm{F}}-E_{\mathrm{V}}$, work function $\varphi$, and ionization energy $\Phi$. Fig. 7a and b presents the magnification of the high energy cut-off and low energy onset of the UPS spectrum recorded for samples $1 \mathrm{c}$ and $4 \mathrm{c}$. The intercept of the background line with a linear approximation of high energy cut-off taken between $1 / 3$ and $2 / 3$ of the cut-off's maximum intensity allows the determination of the surface work function of examined samples according to ${ }^{51}$

$$
\varphi=h \nu-E_{\text {cut-off }}
$$



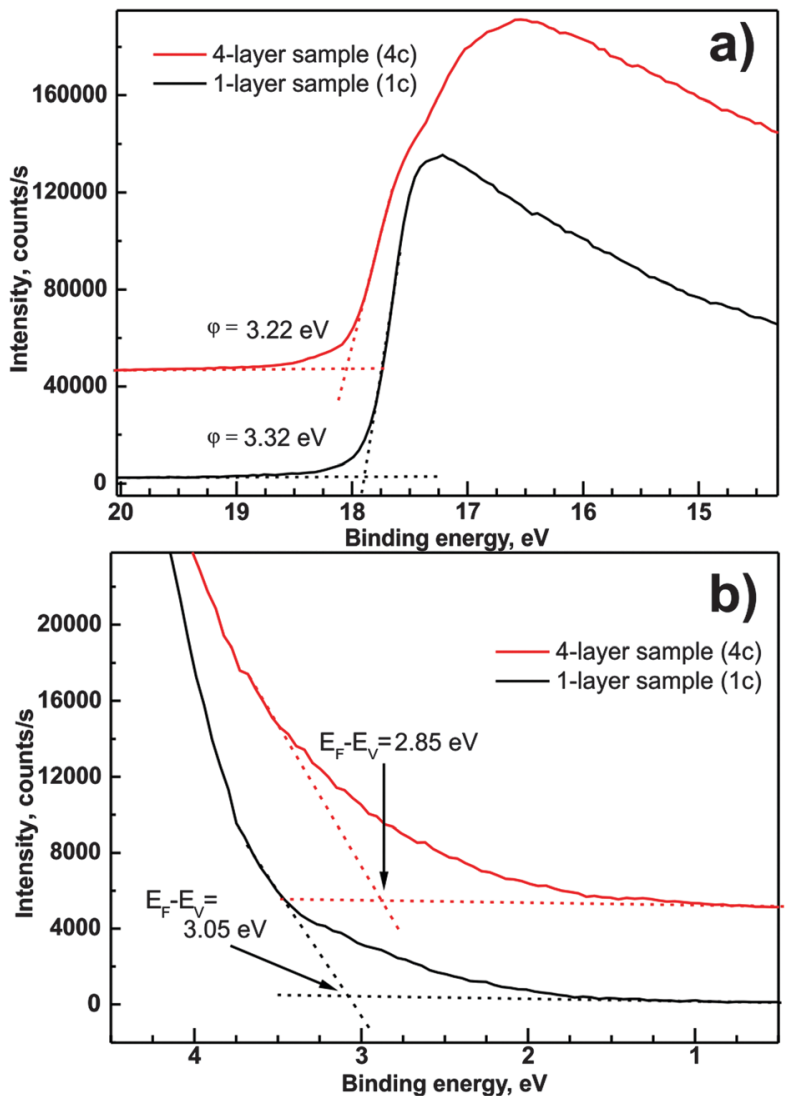

Fig. 7 High energy cut-off (a) and valence band onset (b) of UPS spectra recorded for samples $1 \mathrm{c}$ and $4 \mathrm{c}$.

where $h \nu$ is the excitation energy (here $21.22 \mathrm{eV}$ ) and $E_{\text {cut-off }}$ is the interception point. The obtained work function values were $\varphi=3.32 \mathrm{eV}$ and $\varphi=3.18 \mathrm{eV}$ (uncertainty $0.05 \mathrm{eV}$ ) for samples $1 \mathrm{c}$ and $4 \mathrm{c}$, respectively. Next, the ionization energy was determined as ${ }^{51}$

$$
\Phi=\varphi+\left(E_{\mathrm{F}}-E_{\mathrm{V}}\right)
$$

where $E_{\mathrm{F}}-E_{\mathrm{V}}$ is the energy difference between the valence band onset and the Fermi level position (Fig. 7b). The values of $E_{\mathrm{F}}-E_{\mathrm{V}}$ were determined to be $3.05 \mathrm{eV}$ and $2.85 \mathrm{eV}$ for samples $1 \mathrm{c}$ and $4 \mathrm{c}$, respectively. Hence, the ionization energy is equal to $6.37 \mathrm{eV}$ for $1 \mathrm{c}$ and $6.07 \mathrm{eV}$ (uncertainty $0.07 \mathrm{eV}$ ) for the $4 \mathrm{c}$ sample.

The values of the work function are considerably smaller than $3.74 \mathrm{eV}$ obtained on layers grown by radio frequency magnetron sputter deposition. ${ }^{52}$ On the other hand, our values are quite consistent with the values obtained for (0001)ZnO Zn-terminated surfaces annealed at $700 \mathrm{~K}^{53}$

With this input from the UPS data, the depth dependent XPS measurements from the valence band region were analyzed. $E_{\mathrm{F}}-E_{\mathrm{V}}$ values were determined from examined samples as a function of layer thickness. Simultaneously, the $\mathrm{Zn} 3 \mathrm{~d}$ peak position was analyzed.

Fig. 8 presents high-resolution valence band spectra recorded for sample $4 \mathrm{c}$ for different etching depths. The $\mathrm{Zn}$ $3 \mathrm{~d}$ XPS peak is well visible as well as the valence band onset.
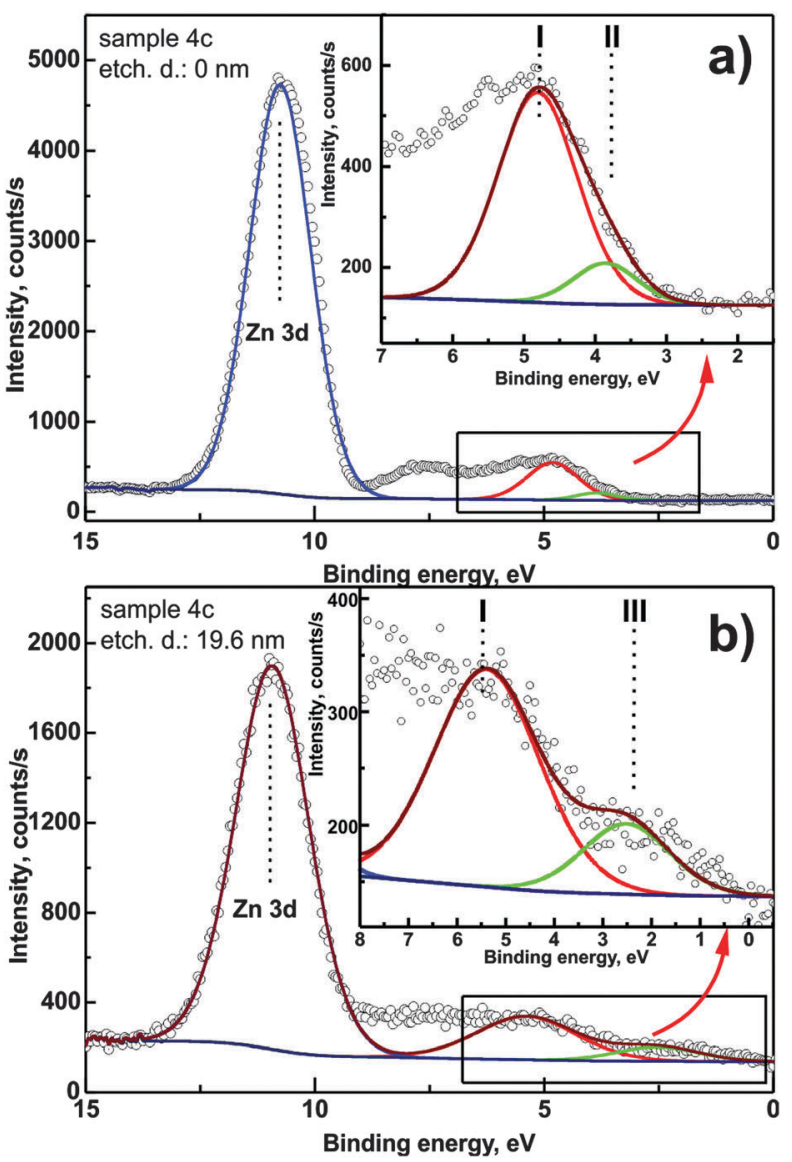

Fig. 8 Valence band region and Zn 3d XPS peaks recorded before etching (a) and after etching $19.6 \mathrm{~nm}$ (b). Insets: magnification of the valence band onset. Two components are distinguishable in each case. For component (I, II and III) description, see the text.

The region of energies between 6 and $8 \mathrm{eV}$ corresponds to $\mathrm{O} 2 \mathrm{p}$, C 2p and Si 3s levels and due to their surface sensitivity in the XPS method they are not considered for further analysis. A binding energy of 0 corresponds to the Fermi level position. It can be clearly seen in the spectra that the onset of photoemission consists of more than one component. For a direct quantification, the band needs to be decomposed. In the presented spectra, three distinct contributions can be observed (marked as I, II and III in Fig. 8). Component (II) is detectable up to an etching depth of $15 \mathrm{~nm}$ and is attributed to defect-related energy levels in the band gap of the zinc oxide layer. Component (III) is related to the substrate-induced distortions of the $\mathrm{ZnO}$ electronic structure.

Each of the components introduces distortions of the energy difference $E_{\mathrm{F}}-E_{\mathrm{V}}$. The $E_{\mathrm{F}}-E_{\mathrm{V}}$ values as a function of ion etching depth determined for the three components are presented in Fig. 9. Dependent on the depth that is probed by XPS, the mutual relation of the components affects the overall $E_{\mathrm{F}}-E_{\mathrm{V}}$ energy difference. The synergy effect can be described using an equivalent component for the valance band representation. Hence we construct such a component with the area equal to the sum of the particular component areas and position which is the weighted mean of the respective positions (we use components I, II and III areas as weights). The onset of 


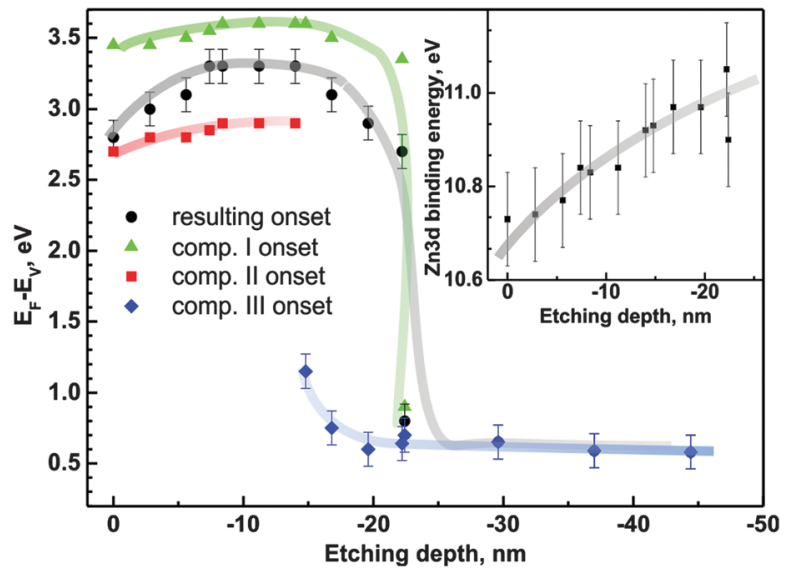

Fig. $9 E_{\mathrm{F}}-E_{\mathrm{V}}$ as a function of ion etching depth (sample 4c). Inset: the $\mathrm{Zn}$ $3 d$ XPS peak position as a function of the depth of ion etching.

the equivalent component determines the resulting $E_{\mathrm{F}}-E_{\mathrm{V}}$ energy distance (full black circles in Fig. 9).

The resulting $E_{\mathrm{F}}-E_{\mathrm{V}}$ value changes from $2.8 \mathrm{eV}$ to $3.3 \mathrm{eV}$ in the range of the first $18 \mathrm{~nm}$ from the sample surface. The component (II) contribution is detectable up to $15 \mathrm{~nm}$ from the surface, while component (III) starts to appear in the spectra from $15 \mathrm{~nm}$. Component (I) is present from the initial surface up to $\sim 22 \mathrm{~nm}$. At this depth, the resultant $E_{\mathrm{F}}-E_{\mathrm{V}}$ value drops significantly to the value of $0.6 \mathrm{eV}$ consistent with the weakly doped n-type silicon substrate (in comparison to $0.56 \mathrm{eV}$ for intrinsic $\mathrm{Si}^{54}$ ). The overall thickness obtained in this ion etching experiment is consistent with the thickness obtained by ellipsometry. The analogous analysis performed for sample 1c shows an initial increase of $E_{\mathrm{F}}-E_{\mathrm{V}}$ from $3.0 \mathrm{eV}$ at the surface to $3.2 \mathrm{eV}$ at the depth of $\sim 4 \mathrm{~nm}$ (not shown in the figure). Then the value stabilizes and drops rapidly down to the substrate-related value of $0.6 \mathrm{eV}$. The changes in the electronic structure occur at similar depths as changes in the $\mathrm{Zn} / \mathrm{O}$ ratio are observed (Fig. $6 \mathrm{~b}$ ).

The inset of Fig. 9 shows the $\mathrm{Zn} 3 \mathrm{~d}$ peak position as a function of depth. The changes, within the accuracy of the method, follow the valence band position changes with respect to the Fermi level.

For a comprehensive description of the depth-dependent ZnO electronic structure, determination of the vacuum level as a function of ion etching depth is necessary. For this purpose, SKP measurements were performed. Fig. 10 shows the change in the contact potential difference (CPD) between the SKP tip and the $4 \mathrm{c}$ sample surface. The measurement was done on the crater etched by the $\mathrm{Ar}^{+}$ions during XPS ion etching depth profiling. The tip of the SKP probed the edge of the crater from an intact layer towards the silicon substrate. Although the obtained potential values are given with respect to a different reference than those obtained using the photoemission methods, the voltage differences are equal to changes in the work function $\Delta \varphi$, i.e. the energy difference between the Fermi level $E_{\mathrm{F}}$ and the vacuum level $E_{\mathrm{V}}$. For sample $4 \mathrm{c}, \Delta \varphi$ equals $0.20 \mathrm{eV}$ with the trend following the $E_{\mathrm{V}}$ and $\mathrm{Zn} 3 \mathrm{~d}$ changes. The observed difference shows a drop in the vacuum level which causes a lowering of the work function towards deeper regions in the $\mathrm{ZnO}$ layers. The same analysis made for sample 1c (not shown) revealed $\Delta \varphi \sim$ $0.10 \mathrm{eV}$. The SKP analysis was followed by carrier concentration calculations. We determined the value of negative carrier concentration $n$ in $\mathrm{ZnO}$ as ${ }^{55}$

$$
n=N_{\mathrm{c}} \cdot\left(\frac{E_{\mathrm{F}}-E_{\mathrm{c}}}{k T}\right)
$$

where $N_{\mathrm{c}}$ is the effective density of states in the conduction band, for $\mathrm{ZnO} N_{\mathrm{c}}=3.7 \times 10^{18} \mathrm{~cm}^{-3},{ }^{56} \mathrm{k}$ is the Boltzmann constant and $T$ is the absolute temperature, here $300 \mathrm{~K}$. The energetic distance, $E_{\mathrm{F}}-E_{\mathrm{c}}$, is calculated as the difference between the $\mathrm{ZnO}$ band gap $E_{\mathrm{g}}=3.37 \mathrm{eV}^{33}$ and the value of $E_{\mathrm{F}}-E_{\mathrm{V}}$ received from XPS measurements of the valence band region.

Fig. 10 shows depth-dependent carrier concentration for samples 1c (full circles) and 4c (triangles). The carrier concentration graph shows significant differences between samples $1 \mathrm{c}$ and $4 \mathrm{c}$. In the case of sample 1c, the carrier concentration changes within the first 3-4 $\mathrm{nm}$ starting from the sample surface and then stabilizes at a level of $10^{16} \mathrm{~cm}^{-3}$. The depth levels at which changes are observed agree with those in the $\mathrm{Zn} /$ $\mathrm{O}$ ratio plot (Fig. 6b). Sample 4c exhibits a gradual carrier concentration change within the first $10 \mathrm{~nm}$ and then stabilizes at the level of $10^{18} \mathrm{~cm}^{-3}$. The drop in the carrier concentration for the last point is due to layer-substrate interaction and discussed earlier as caused by signal overlap of the XPS signals used for calculating the values presented here. The data in Fig. 10 highlight the differences between samples $1 \mathrm{c}$ and $4 \mathrm{c}$ in chemical composition and electronic properties. It also clarifies the differences between regions near the surface and regions inside the thin film for both samples.

All data determined above were gathered and utilized to construct a band-like energy structure diagram of spin-coated ZnO layers which is shown in Fig. 11. The diagram was drawn

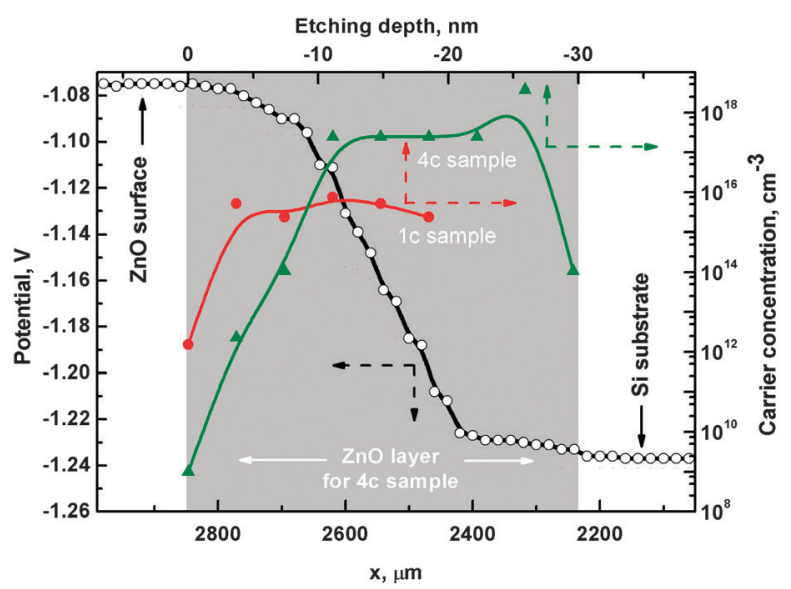

Fig. 10 SKP profile (empty circles - left-bottom scale) of sample 4c. The abscissa corresponds to the position on the edge of the ion etched crater in the $\mathrm{ZnO}$ layer. The ordinates represent the potential difference between the tip and the sample surface. Concentration of $n$-type charge carriers (right-top scale) is shown as a function of ion etching depth for samples $1 \mathrm{c}$ (full circles) and $4 c$ (triangles). Dashed arrows additionally indicate the corresponding axis. 
for sample $4 \mathrm{c}$, because the depth-dependent chemical and energy changes are not so pronounced in the case of thinner, more uniform and compact sample 1c. Analyzing the energy levels in the $\mathrm{ZnO}$ layer, three different regions can be distinguished. They are denoted, starting from the surface side moving towards the substrate, as $\mathrm{n}, \mathrm{n}^{+}$, and oxide/substrate interface. The naming of the first two regions originates from different electronic properties describing zinc oxide in that particular region.

Starting from the surface, the $\mathrm{n}$ region extends $10 \mathrm{~nm}$ deep into the $\mathrm{ZnO}$ layer. Within this region, the energy levels are altered, which is manifested by the significant $(\sim 0.5 \mathrm{eV})$ band bending of the valence and conduction bands in comparison to the next region, denoted as $\mathrm{n}^{+}$. The $\mathrm{Zn} 3 \mathrm{~d}$ core level follows this trend. With the band bending, the work function decreases by $0.2 \mathrm{eV}$.

Within the $n$ region, there is a considerable variation of the $\mathrm{Zn} / \mathrm{O}$ ratio from 1.7 to 2.1 , as oxygen uptake occurs from the surface towards the interior of the $\mathrm{ZnO}$ layer. Oxygen from the ambient atmosphere may be incorporated into the layer from the surface region (n) during annealing. Alternatively, water vapor may adsorb after annealing. The defects introduce additional states in the band gap below the Fermi level, which alter the valence band position and consequently $E_{\mathrm{F}}-E_{\mathrm{V}}$. They also improve stoichiometry at the sample surface reducing the $\mathrm{Zn} / \mathrm{O}$ ratio.

The $\mathrm{n}^{+}$region is characterized by a significantly higher carrier concentration and the relatively small separation between the Fermi level $E_{\mathrm{F}}$ and the bottom of the conduction band $E_{\mathrm{C}}$. In this region, $\mathrm{ZnO}$ tends towards a metal-like electronic structure. The origin of this variation is most likely the increase of oxygen

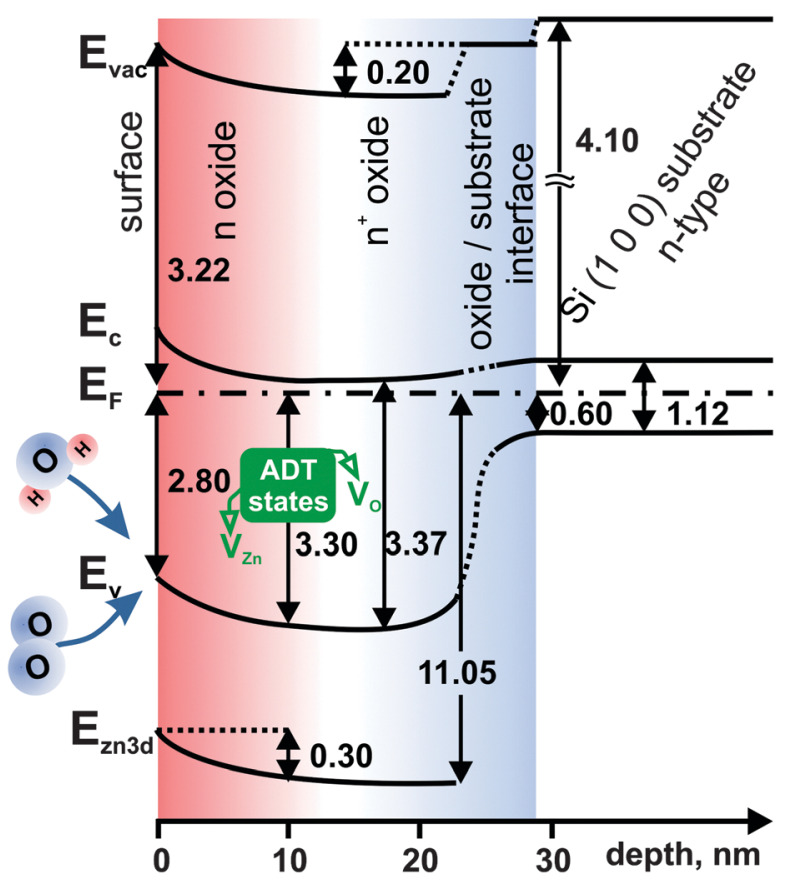

Fig. 11 Band-like diagram of the electronic structure of deposited ZnO layers for sample 4c. The Si work function is from ref. 57 and the Si band gap from ref. 58. The work function change at the oxide/substrate interface can be interpreted as the interface dipole effect. ${ }^{59}$ Acceptor-donor-trap states alter the energy levels. vacancy concentration towards the interior region of the zinc oxide film.

Looking at the direction of the band bending, one expects charge carrier depletion in the $\mathrm{n}$ region. Recalling the results shown in Fig. 10 one can see that excess carrier concentration is considerably lower in the $\mathrm{n}$ than the $\mathrm{n}^{+}$region.

However it has to be remembered that the photoemission signal, which is the main basis of the constructed diagram, comes from the so-called XPS information depth, which can be up to $10 \mathrm{~nm}$. In these terms the different energetic states have their contribution to the overall photoemission signal. It means that the states decreasing the carrier concentration (states rather close to the surface) and states increasing the carrier concentration (more distant from the surface and bulk-related) are simultaneously observed in photoemission spectra giving the combined effect. ${ }^{60}$ The states are characterized by different intensities. Consequently, the states which are present in the ZnO spectrum must have a competitive nature. ${ }^{61}$ On the one hand, the states should be of donor-type due to increased accumulation in the $\mathrm{n}^{+}$region, while on the other hand, lowering the number of excess carriers in the $n$ region suggests the existence of states with acceptor-like or trap nature.

A possible explanation for this situation is considering a pairing of oxygen and zinc vacancies ( $\mathrm{V}_{\mathrm{O}}$ and $\mathrm{V}_{\mathrm{Zn}}$ respectively). Although the layer is strongly oxygen deficient throughout, with the increase of oxygen deficiency towards the layer interior, the competing zinc vacancies introduce the acceptor states. Simultaneously, due to the existence of $\mathrm{V}_{\mathrm{O}}$ and $\mathrm{V}_{\mathrm{Zn}}$ together, the native $\mathrm{ZnO}$ structure has to be significantly deformed. This deformation might introduce additional trap levels which alter the photoemission experiment, indirectly changing the carrier concentration distribution pattern. Hence, from the experimental point of view, at the surface, three different and mutually competing energy states are present as one group. Therefore, we propose to treat them as one group of acceptordonor-trap states (referred to as "ADT" states).

The individual states of different nature were described with their energy levels. ${ }^{62}$ As a result, $\mathrm{Zn}$ vacancies should be treated as deep acceptors with the three possible charge state configurations, $\mathrm{V}_{\mathrm{Zn}}^{0}, \mathrm{~V}_{\mathrm{Zn}}^{-}$and $\mathrm{V}_{\mathrm{Zn}}^{2-} \cdot \mathrm{V}_{\mathrm{Zn}}$ has the lowest formation energy among the native point defects in $\mathrm{ZnO}$; hence it is expected to be the dominant point defect class. Generally, the absence of a $\mathrm{Zn}$ atom in the $\mathrm{ZnO}$ lattice should leave four oxygen dangling bonds with six electrons. As stated in ref. 33, dangling bonds can combine into doubly occupied symmetric states located deep in the valence band, and three almost degenerate states in the band gap close to the top of the valence band. In our situation, the latter states are the center of attention, because the states can easily accept two additional electrons (they are partially occupied by four electrons ${ }^{33}$ ) altering the electronic structure in the band gap. The ADT state donor part is connected with the oxygen vacancies. Such vacancies have, among the donor defects, the lowest formation energy. ${ }^{33}$ This low formation energy is caused by the Madelung potential, which is the main driving force for the localization of electrons in the place normally occupied by the oxygen anion in the $\mathrm{ZnO}$ 
structure. The replacement of oxygen by two free electrons in the defective lattice minimizes the energetic cost of the vacancy formation. ${ }^{63}$ Free electrons have been observed in $\mathrm{ZnO}$ experimentally by electron paramagnetic resonance. ${ }^{64}$ States associated with these electrons should act as shallow donors. ${ }^{61,65,66}$ On the other hand, density functional calculations ${ }^{67}$ have shown that $V_{O}$ leads to very deep donor states which cannot contribute to n-type conductivity. This statement is not consistent with our results. However, because the neutral $\mathrm{V}_{\mathrm{O}}$ is located at $\sim 1 \mathrm{eV}$ below the bottom of the conduction band, ${ }^{30}$ it can compete with the nearby acceptor states $\mathrm{V}_{\mathrm{Zn}}$ during photoexcitation of the sample affecting the photoemission experiment. Ionized vacancies like $\mathrm{V}_{\mathrm{O}}^{+}$are also deep donors, ${ }^{68,69}$ but according to the literature, $\mathrm{V}_{\mathrm{O}}^{+}$is not thermodynamically stable. ${ }^{70}$

The state originating from the coordinatively unsaturated anion $\mathrm{O}^{2-}$ (ref. 71) may also be present as a donor state. Such anions may be introduced by the environment, e.g. from $\mathrm{O}_{2}$ or water vapor.

The last component of the ADT states that remains to be discussed is the traps located in the band gap of ZnO. Such electron traps significantly affect the results of the photoemission experiment, and consequently the calculation of free carriers. The most probable are shallow electron traps $\mathrm{ZnO}_{\text {corner }}\left(\mathrm{e}^{-}\right)$at morphological features like surface inhomogeneity. The more structured surface with column-like features observed for sample 4c (Section 3.1) hints in this direction, as less traps are expected for samples $1 \mathrm{c}$ with a more uniform surface. Similar morphology-related trap states were observed earlier. ${ }^{72}$ Moreover, electrons can be entrapped in a localized cavity center of a missing $\mathrm{O}$ atom either from the interior region or from the surface of $\mathrm{ZnO} .^{33}$ Hence, oxygen vacancies may be responsible for the observation of trap states.

\section{Conclusions}

The presented work shows preparation and characterization of extremely oxygen-deficient low-work function zinc oxide layers with a gradient of carrier concentration. The preparation uses an easy and cost-effective sol-gel process followed by spin-coating. Surface morphology can be tuned by the number of deposition cycles. Such layers can be applied in bulk heterojunction-based photovoltaic devices.

The sol-gel preparation technique permits control over the carrier concentration profile from the subsurface region towards the interior film depending on particular needs. This work demonstrates two radically different films: one with a significant carrier concentration gradient and the other with a homogeneous carrier distribution.

The observed band bending, together with the information on the composition, leads to the conclusion that several types of defects combine their properties to give a group with donor, acceptor and trap properties, which cannot be treated independently. The origin of such "acceptor-donor-trap" (ADT) states may be oxygen and zinc vacancies, morphology-induced trap states and surface states resulting from the interaction with an ambient atmosphere.
The sol-gel preparation technique offers still much wider opportunities for preparation than explored in this work. For instance, the column-like morphology observed for some films makes them candidates for applications in photovoltaic and nanoelectronic device preparation, e.g. in hybrid inorganic-organic electronic devices with p-type phthalocyanines. Addition of foreign components into the solution offers the opportunity to tune doping levels more precisely than shown here. While in gas phase processes, the variation of composition of a thin film is more straightforward to realize, the ease of solution techniques makes it worthwhile to take up the challenge of unraveling the interplay between preparation and electronic properties of the resulting films. The result may be a knowledge-based design with completely new properties in long-known materials.

\section{Acknowledgements}

This scientific work was partially supported by the Polish budget for science in years 2013-2015 (by Ministry for Science and Higher Education) within the Iuventus Plus IP2012 019072 project through the Silesian University of Technology, Institute of Physics.

\section{References}

1 J. B. Webb, D. F. Williams and M. Buchanan, Appl. Phys. Lett., 1981, 39, 640.

2 M. J. Brett, R. W. McMahon, J. Affinito and R. R. Parsons, J. Vac. Sci. Technol., A, 1983, 1, 352.

3 H. S. Uhm, G. C. Kwon and E. H. Choi, Appl. Phys. Lett., 2011, 99, 261502.

4 Y. Hagiwara, T. Nakada and A. Kunioka, Sol. Energy Mater. Sol. Cells, 2001, 67, 267.

5 D. R. Kumar, D. Manoj and J. Santhanalakshmi, RSC Adv., 2014, 4, 8943.

6 C. Battaglia, J. Escarré, K. Söderström, M. Charrière, M. Despeisse, F.-J. Haug and C. Ballif, Nat. Photonics, 2011, 5, 535.

7 L. Xu, X. Li, Y. Chen and F. Xu, Appl. Surf. Sci., 2011, 257, 4031.

8 S. S. Badadhe and I. S. Mulla, Sens. Actuators, B, 2009, 143, 164.

9 D. Zappa, E. Comini and G. Sberveglieri, Nanotechnology, 2013, 24, 444008.

10 R. Moirangthem and A. Erbe, Appl. Phys. Lett., 2013, 103, 051108.

11 M. Willander, O. Nur, N. Bano and K. Sultana, New J. Phys., 2009, 11, 125020.

12 F.-L. Kuo, Y. Li, M. Solomon, J. Du and N. D. Shepherd, J. Phys. D: Appl. Phys., 2012, 45, 065301.

13 C. L. Jia, K. M. Wang, X. L. Wang, X. J. Zhang and F. Lu, Opt. Express, 2005, 13, 5093.

14 Z. H. Chen, C. P. Liu, H. E. Wang, Y. B. Tang, Z. T. Liu, W. J. Zhang, S. T. Lee, J. A. Zapien and I. Bello, J. Phys. D: Appl. Phys., 2011, 44, 325108. 
15 C. Reese, M. Roberts, M. M. Ling and Z. Bao, Mater. Today, 2004, 7, 20.

16 A. L. Briseno, T. W. Holcombe, A. I. Boukai, E. C. Garnett, S. W. Shelton, J. J. M. Frechet and P. Yang, Nano Lett., 2010, 10, 334 .

17 Y. Natsume, H. Sakata, T. Hirayama and H. Yanagida, J. Appl. Phys., 1992, 72, 4203.

18 M. Chandran, B. Tiwari, C. R. Kumaran, S. K. Samji, S. S. Bhattacharya and M. S. Ramachandra Rao, J. Phys. D: Appl. Phys., 2012, 45, 202001.

19 T. Chen, S.-L. Liu, Q. Xie, C. Detavernier, R. L. Van Meirhaeghe and X.-P. Qu, J. Mater. Sci.: Mater. Electron., 2010, 21, 88.

20 M. A. Pietrzyk, M. Stachowicz, A. Wierzbicka, P. Dluzewski, D. Jarosz, E. Przeździecka and A. Kozanecki, J. Cryst. Growth, 2014, 408, 102.

21 K. B. Sundaram and A. Khan, Thin Solid Films, 1997, 295, 87. 22 X. Jiang, F. L. Wong, M. K. Fung and S. T. Lee, Appl. Phys. Lett., 2003, 83, 1875.

23 C. Besleaga, G. E. Stan, A. C. Galca, L. Ion and S. Antohe, Appl. Surf. Sci., 2012, 258, 8819.

24 D. Bekermann, et al., Cryst. Growth Des., 2010, 10, 2011-2018.

25 K. L. Narasimhan, S. P. Pai, V. R. Palkar and R. Pinto, Thin Solid Films, 1997, 295, 104.

26 Y. Chen, P. Schneider, B.-J. Liu, S. Borodin, B. Ren and A. Erbe, Phys. Chem. Chem. Phys., 2013, 15, 9812.

27 D. Iqbal, A. Kostka, A. Bashir, A. Sarfraz, Y. Chen, A. D. Wieck and A. Erbe, ACS Appl. Mater. Interfaces, 2014, 6, 18728.

28 X. Liu and M. T. Swihart, Nanoscale, 2013, 5, 8029.

29 X. Tang, E. S. Guang Choo, L. Li, J. Ding and J. Xue, Chem. Mater., 2010, 22, 3383.

30 Y. Natsume and H. Sakata, Thin Solid Films, 2000, 372, 30.

31 L. Xu, X. Li, Y. Chen and F. Xu, Appl. Surf. Sci., 2011, 257, 4031.

32 A. H. Jayatissa, K. Guo, T. Gupta and A. C. Jayasuriya, J. Mater. Sci.: Mater. Electron., 2009, 20, 577.

33 A. Janotti and C. G. Van de Walle, Rep. Prog. Phys., 2009, 72, 126501.

34 A. Ney, M. Opel, T. C. Kaspar, V. Ney, S. Ye, K. Ollefs, T. Kammermeier, S. Bauer, K.-W. Nielsen, S. T. B. Goennenwein, M. H. Engelhard, S. Zhou, K. Potzger, J. Simon, W. Mader, S. M. Heald, J. C. Cezar, F. Wilhelm, A. Rogalev, R. Gross and S. A. Chambers, New J. Phys., 2010, 12, 013020.

35 M. Krzywiecki, L. Grządziel, J. Bodzenta and J. Szuber, Thin Solid Films, 2012, 520, 3965.

36 M. Krzywiecki, L. Grządziel, J. Juszczyk, A. Kaźmierczak-Bałata, A. Erbe and J. Bodzenta, J. Phys. D: Appl. Phys., 2014, 35, 335304.

37 H. R. Kerp, H. Donker, R. B. M. Koehorst, T. J. Schaafsma and E. E. van Faassen, Chem. Phys. Lett., 1998, 298, 302.

38 http:/gwyddion.net/documentation/user-guide-en/.

39 I. Lindau, P. Pianetta, K. Y. Yu and W. E. Spicer, Phys. Rev. B: Solid State, 1976, 16, 492.

40 J. Blochwitz, T. Fritz, M. Pfeiffer, K. Leo, D. M. Alloway, P. A. Lee and N. R. Armstrong, Org. Electron., 2001, 2, 97.

41 M. Chandran, B. Tiwari, C. R. Kumaran, S. K. Samji, S. S. Bhattacharya and M. S. Ramachandra Rao, J. Phys. D: Appl. Phys., 2012, 45, 202001.
42 R. Yogamalara, R. Srinivasan, A. Vinu, K. Ariga and A. C. Bose, Solid State Commun., 2009, 149, 1919.

43 H. P. Klug and L. E. Alexander, X-Ray Diffraction Procedures, John Wiley \& Sons Inc., 1974, 2nd edn, pp. 687-703.

44 www.lasurface.com/database/element.php.

45 M. Futsuhara, K. Yoshioka and O. Takai, Thin Solid Films, 1998, 322, 274.

46 L. Pauling, The Nature of the Chemical Bond, Cornell University, Ithaca, NY, 1960, ch. 3.

47 M. Futsuhara, K. Yoshioka and O. Takai, Thin Solid Films, 1998, 322, 274.

48 A. S. Komolov, S. N. Akhremtchik and E. F. Lazneva, Spectrochim. Acta, Part A, 2011, 79, 708.

49 P. Erhart, A. Klein and K. Albe, Phys. Rev. B: Condens. Matter Mater. Phys., 2005, 72, 085213.

50 M. P. Seah and W. A. Dench, Surf. Interface Anal., 1979, 1, 2. 51 P. Y. Yu and M. Cardona, Fundamentals of SemiconductorsPhysics and Materials Properties, Springer-Verlag, Berlin Heidelberg, 2001.

52 F.-L. Kuo, Y. Li, M. Solomon, J. Du and N. D. Shepherd, J. Phys. D: Appl. Phys., 2012, 45, 065301.

53 K. Jacobi, G. Zwicker and A. Gutmann, Surf. Sci., 1984, 141, 101. 54 H. F. Wolf, Semiconductors, J. Wiley \& Sons Inc., New York, 1971.

55 W. Monch, Semiconductor Surfaces and Interfaces, SpringerVerlag, Berlin, 1995.

56 C. Maragliano, S. Lilliu, M. S. Dahlem, M. Chiesa, T. Souier and M. Stefancich, Sci. Rep., 2014, 4, 4203.

57 S. N. Novikov and S. P. Timoshenkov, Russ. J. Phys. Chem. A, 2010, 84, 1266.

58 B. Pellegrini, J. Phys. D: Appl. Phys., 1976, 9, 55.

59 H. Lüth, Surfaces and Interfaces of Solid Materials, Springer-Verlag, Berlin, 1995.

60 B. Saha, N. S. Das and K. K. Chattopadhyay, Thin Solid Films, 2014, 562, 37.

61 A. Weidinger, J. M. Gil, H. V. Alberto, R. C. Vilao, J. Piroto Duarte, N. Ayres de Campos and S. FJ. Cox, Physica B, 2003, 326, 124.

62 A. Janotti and C. G. Van de Walle, Phys. Rev. B: Condens. Matter Mater. Phys., 2007, 75, 165202.

63 G. Pacchioni, ChemPhysChem, 2003, 4, 1041.

64 S. Polarz, Angew. Chem., Int. Ed., 2006, 45, 2965.

65 D. Seghier and H. P. Gisalson, J. Mater. Sci.: Mater. Electron., 2008, 19, 687.

66 B. Monemar, U. Lindefelt and W. M. Chen, Physica B, 1987, 146, 256.

67 A. Janotti and C. G. Van de Walle, J. Cryst. Growth, 2006, 287, 58. 68 S. Lany and A. Zunger, Phys. Rev. Lett., 2007, 98, 045501.

69 S. Lany and A. Zunger, Phys. Rev. B: Condens. Matter Mater. Phys., 2005, 72, 035215.

70 A. Janotti and C. G. Van de Walle, Appl. Phys. Lett., 2005, 87, 122102.

71 G. Pacchioni, J. M. Ricart and F. Illas, J. Am. Chem. Soc., 1994, 116, 10152.

72 P. V. Sushko, J. L. Gavartin and A. L. Shluger, J. Phys. Chem. $B, 2002,106,2269$. 